\title{
Effects of high-dose fish oil supplementation during early infancy on neurodevelopment and language: a randomised controlled trial
}

\author{
Suzanne J. Meldrum ${ }^{1,2 *}$, Nina D’Vaz ${ }^{1}$, Karen Simmer $^{1,2}$, Janet A. Dunstan ${ }^{1}$, Kathryn Hird ${ }^{1,3}$ \\ and Susan L. Prescott ${ }^{1}$ \\ ${ }^{1}$ School of Paediatrics and Child Health, University of Western Australia, M561, 35 Stirling Highway, Crawley, 6009 WA, \\ Australia \\ ${ }^{2}$ School of Women's and Infants Health, University of Western Australia, M550, 35 Stirling Highway, Crawley, 6009 WA, \\ Australia \\ ${ }^{3}$ Faculty of Medicine, Notre Dame University, PO Box 1225, WA 6959, Australia \\ (Submitted 31 May 2011 - Final revision received 2 November 2011 - Accepted 17 November 2011 - First published online 21 February 2012)
}

\section{Abstract}

$n$-3 Long-chain PUFA (LC-PUFA) intake during infancy is important for neurodevelopment; however, previous studies of $n$-3 LC-PUFA supplementation have been inconclusive possibly due to an insufficient dose and limited methods of assessment. The present study aimed to evaluate the effects of direct supplementation with high-dose fish oil (FO) on infant neurodevelopmental outcomes and language. In the present randomised, double-blind, placebo-controlled trial, 420 healthy term infants were assigned to receive a DHA-enriched FO supplement (containing at least $250 \mathrm{mg} \mathrm{DHA} / \mathrm{d}$ and $60 \mathrm{mg} \mathrm{EPA} / \mathrm{d}$ ) or a placebo (olive oil) from birth to 6 months. Assessment occurred at 18 months via the Bayley Scales of Infant and Toddler Development (3rd edition; BSID-III) and the Child Behavior Checklist. Language assessment occurred at 12 and 18 months via the Macarthur-Bates Communicative Development Inventory. The FO group had significantly higher erythrocyte DHA $(P=0.03)$ and plasma phospholipid DHA $(P=0 \cdot 01)$ levels at 6 months of age relative to placebo. In a small subset analysis (about $40 \%$ of the total population), children in the FO group had significantly higher percentile ranks of both later developing gestures at 12 and 18 months $(P=0 \cdot 007 ; P=0 \cdot 002$, respectively) and the total number of gestures $(P=0 \cdot 023 ; P=0 \cdot 006$, respectively). There was no significant difference between the groups in the standard or composite scores of the BSID-III. The results suggest that improved postnatal $n$-3 LC-PUFA intake in the first 6 months of life using high-dose infant FO supplementation was not beneficial to global infant neurodevelopment. However, some indication of benefits to early communicative development was observed.

Key words: $\boldsymbol{n}$-3 Long-chain PUFA: Infant supplementation: Neurodevelopment: Language

$n$-3 and $n$-6 long-chain PUFA (LC-PUFA) including DHA and arachidonic acid (AA) serve as indispensable structural components of cellular membranes and are important for normal retinal and neuronal development ${ }^{(1,2)}$. Infants have a limited conversion of LC-PUFA from its precursor 18-carbon fatty acids (FA), with high dependence on LC-PUFA provided by antenatal placental transfer and later from breast milk or infant formula $^{(3)}$. As maternal LC-PUFA intake is the major determinant of LC-PUFA transfer to the offspring ${ }^{(4)}$, there has been growing concern that the low intakes of $n$-3 LC-PUFA intake in Western nations, particularly DHA, may place infants at risk of deficiency during critical early period of neurological development ${ }^{(5)}$.

The functional effects of $n$-3 LC-PUFA upon outcomes such as visual acuity and other neurodevelopmental markers have been explored in many randomised controlled trials of
LC-PUFA supplementation in term infants ${ }^{(6-28)}$. Such studies have typically evaluated the effects of LC-PUFA-supplemented formula, which historically contained no preformed LC-PUFA. In breast-feeding infants, there have been trials in which lactating mothers were supplemented to increase LC-PUFA levels derived through breast milk ${ }^{(29-32)}$. Both methods, however, have provided conflicting evidence on the question of whether postnatal LC-PUFA supplementation is beneficial to neurodevelopment in term infants ${ }^{(33,34)}$.

There are a number of proposed reasons for the lack of definitive findings in previous research trials. First, previous dosages may have been insufficient to determine differences between treatment groups. Most studies in healthy term infants have raised DHA intakes to approximately that of breast-fed infants in Western nations, between 0.2 and $0.3 \%$

Abbreviations: AA, arachidonic acid; BSID-III, Bayley Scales of Infant and Toddler Development (3rd edition); CBCL, Child Behavior Checklist; FA, fatty acid; FO, fish oil; LC-PUFA, long-chain PUFA; MCDI, Macarthur-Bates Communicative Development Inventory.

*Corresponding author: Dr S. J. Meldrum, fax +619388 2097, email suzanne.meldrum@uwa.edu.au 
FA in breast milk. In contrast, nations such as Japan report breast milk levels of up to $1 \%$, probably due to high fish intake ${ }^{(35)}$. Currently, there have been no randomised controlled trials to evaluate the effects of direct supplementation with high-dose LC-PUFA $(>1 \%)$ in term infants.

Second, there has been a lack of trials that have investigated the effects of directly supplementing infants with $n$-3 LC-PUFA independently from the feeding method. Agostoni et al. ${ }^{(20)}$ in a recent study provided DHA in liquid form during the first year of life, but provided only $20 \mathrm{mg}$, chosen as it would be the minimum amount supplied within human milk. Consequently, there have been no trials that have investigated the effects of directly supplementing infants with n-3 LC-PUFA at higher doses. Direct supplementation could be advantageous, as it is applicable to the entire infant population, regardless of the feeding practice.

Lastly, a frequently proposed reason for inconsistencies in previous findings is the use of scientifically inadequate or insensitive methods for developmental assessment. A large percentage of controlled trials include global tests of development, which are not designed to detect subtle changes in brain function ${ }^{(36)}$. The assessment of language proficiency involving its interdependent aspects of comprehension, gesture, spoken language and appropriate social behaviour, all of which are dependent on integrated neural networks and rapid speed of processing, could be a more suitable domain in which to evaluate nutritional effects on infant development.

To our knowledge, this is the first double-blind, randomised, controlled study to evaluate the effects of fish oil (FO) supplementation for the first 6 months in healthy term infants on neurodevelopment at 18 months, and language development at 12 and 18 months of age. Secondary objectives were to evaluate the effect of FO supplementation on erythrocyte and plasma phospholipid FA at 6 months of age, and associations between FA levels and neurodevelopmental outcomes.

\section{Subjects and methods}

The study design and methodology have been described elsewhere ${ }^{(37)}$. A brief summary is provided below.

\section{Subjects}

The study population included the infants of 420 women in Perth, Western Australia who were recruited for a doubleblind, randomised, controlled study conducted at Princess Margaret Hospital for Children, aimed at assessing the effects of infant FO supplementation on both infant allergy and neurodevelopment. Women were recruited during the third trimester of pregnancy between 1 June 2005 and 1 October 2008 from private and public metropolitan antenatal clinics. Allergic pregnant women were recruited as their infants are at a higher risk of developing allergic disease ${ }^{(38)}$. Maternal atopy was defined by at least one positive skin prick test to at least one of a defined panel of allergens. Exclusion criteria included maternal smoking, a pre-existing medical condition or high-risk pregnancy, more than three fish meals consumed per week or FO intake during pregnancy in excess of
$1000 \mathrm{mg} / \mathrm{d}$, preterm delivery and infants with significant congenital abnormalities or medical conditions.

\section{Study design}

Infants were randomised to receive either a high-dose DHAenriched ethyl ester FO supplement, aimed at delivering 250-280 mg DHA/d ( $n$ 218) or an image-matched placebo containing olive oil ( $n$ 202). The main supplier for the study was Ocean Nutrition, Canada Limited. Capsules were purchased in one batch in 2005, and the composition was regularly tested by an independent laboratory during the trial. The FO capsules contained $650 \mathrm{mg}$ FO comprising $280 \mathrm{mg}$ DHA and $110 \mathrm{mg}$ EPA. The placebo capsules contained $650 \mathrm{mg}$ olive oil (66.6\% n-9 oleic acid). The FA composition of the capsules remained unchanged over the course of the study and peroxide and acid levels remained compliant with Australian standards. Because the trial took longer than anticipated, and because Ocean Nutrition discontinued the original products, the final forty-three children received capsules donated from Numega. These TAG FO capsules contained a similar amount of DHA (260 mg DHA and $60 \mathrm{mg}$ EPA) and the placebo capsules were also olive oil. This brand substitution was endorsed and supervised by the Ethics Committee at Princess Margaret Hospital. Both capsules were image and scent matched. There was no significant difference between the erythrocyte DHA $(P=0.732)$ or plasma phospholipid DHA $(P=0 \cdot 160)$ levels between the two groups of participants receiving FO capsules from different suppliers, so all children were included in the final analysis.

Supplementation commenced at birth and ceased at 6 months of age. Although the primary outcomes were analysed on an 'intention-to-treat' basis, we also examined and allowed for differences in capsule consumption between the groups (see below). Capsule consumption/adherence was based on capsule diaries and a count of the returned capsules, in addition to the infant FA analysis (see below).

Randomisation and the allocation of capsules were completed by external staff (Princess Margaret Hospital Pharmacy) via computer software using an unpredictable allocation sequence, stratified according to maternal atopy, paternal atopic history and parity. Mothers and study personnel were unaware of the group allocation. Intervention and control capsules were image matched, and packaged in identical containers labelled with the participant's name and birth date.

The dose was chosen as a high dosage in comparison with previous trials studying the effects of $n$ - 3 LC-PUFA supplementation in infancy, under the assumption that this would optimise the likelihood of detecting biologically or clinically relevant effects of supplementation. It was recommended that capsules be given to babies in the morning immediately before breast-feeding (capsule pierced and squirted into the infant's mouth), or in the formula during their first daily feed.

Results of the allergic and immunological outcomes will be published elsewhere. Information regarding weight, height, head circumference, relevant medical diagnosis and mode of feeding (including brand of infant formula) was collected at each clinical visit. Demographic and obstetric data were 
collected at birth and participants were seen at 3, 6, 12 and 18 months.

The study was conducted according to the guidelines laid down in the Declaration of Helsinki and all procedures involving human subjects were approved by the Princess Margaret Hospital Research and Ethics Committee. Written informed consent was obtained from all participants.

\section{Fatty acid analysis}

Where possible, two blood samples were collected (1) from cord blood at birth and (2) from infants at age 6 months, following the supplementation period. Phospholipid FA composition was measured in both erythrocyte cell membranes and plasma to provide an indication of FA status over the preceding 6 months ${ }^{(39)}$. Fatty acid compositional analysis was completed as described previously ${ }^{(40)}$. Briefly, peripheral and cord blood erythrocytes were collected into heparinised Roswell Park Memorial Institute (RPMI) medium and cells separated using density centrifugation. Erythrocytes were isolated, washed and stored at $-20^{\circ} \mathrm{C}$ in methanol-chloroform (2:1) and lipids were extracted by TLC. Plasma $(0.5 \mathrm{ml})$ was extracted with chloroform-methanol (2:1, by volume, $5 \mathrm{ml})$ and the phospholipid fraction was obtained from total lipid extracts by TLC. Fatty acid methyl esters were prepared by treating phospholipid extracts with $4 \% \mathrm{H}_{2} \mathrm{SO}_{4}$ (by volume) in methanol at $100^{\circ} \mathrm{C}$ for $10 \mathrm{~min}$ and analysed by GLC on an Agilent 7890 Series Gas Chromatograph System equipped with an Agilent 7683 Autosampler and an Agilent $7683 \mathrm{~B}$ Autoinjector Module (Agilent Technologies Australia Private Limited). Chromatography was performed on a Supelco SP-2560 column $(100 \mathrm{~m} \times 0.25 \mathrm{~mm} \times 0.20 \mu \mathrm{m}$; Sigma-Aldrich Private Limited) using hydrogen as the carrier gas at a split ratio of $30: 1$. The temperature programme was $200^{\circ} \mathrm{C}$ for $8.5 \mathrm{~min}$, then $8{ }^{\circ} \mathrm{C} / \mathrm{min}$ to $240{ }^{\circ} \mathrm{C}$ and held for $4.5 \mathrm{~min}$. Peaks were identified by comparing them with a known standard mixture. Individual FA were calculated as a relative percentage with the evaluated FA set at $100 \%$.

\section{Breast milk fatty acid analysis}

Maternal breast milk samples were collected at 3 and 6 months of age either by manual expression or by breast pump. They were frozen immediately and stored at $-80^{\circ} \mathrm{C}$ until analysis. Fatty acid analysis was performed on the thawed whole milk samples as described previously ${ }^{(41)}$. Briefly, lipids were extracted and transmethylated using $1 \% \mathrm{H}_{2} \mathrm{SO}_{4}$ in methanol. Fatty acid methyl esters were separated using a gas chromatograph with flame ionisation detection using a modification of the previously published method ${ }^{(42)}$. Fatty acids were identified based on retention time to authentic lipid standards and expressed as mean percentages and standard deviations of the total FA measured.

\section{2-month assessment}

Macarthur-Bates Communicative Development Inventories (Words and Gestures) (MCDI) ${ }^{(43)}$ (see below) were posted to the parents of the infants to complete before the 12month clinical visit for allergy.

\section{8-month assessment}

Participants were invited to attend a neurodevelopmental appointment at 18 months of age. The assessments were completed by two assessors (rater 1: $n$ 50, rater 2: $n$ 237) trained in the administration of the tests in a quiet room. The following assessments were completed:

(1) Bayley Scales of Infant and Toddler Development (3rd edition; BSID-III) ${ }^{(44)}$ is an internationally recognised tool to assess the development in very young children (1-42 months). It consists of three scales administered with child interaction including cognitive, motor (consisting of fine and gross motor skill) and language (consisting of expressive and receptive language); and two scales conducted with parent questionnaires including social-emotional and adaptive behaviour. Raw scores of successfully completed items are converted to scale scores (10 (SD 3)) and to composite scores (100 (SD 15)).

(2) Achenbach Child Behavior Checklist (CBCL) ${ }^{(45)}$ was used to assess mental health and behavioural development. It measures parental perceptions of child competencies and behaviours in children aged 18 months to 5 years. Scales include affective problems, anxiety problems, attention deficit/hyperactivity problems, oppositional defiant problems and pervasive developmental problems. $T$-scores are obtained for each of these scales based on the number and extent of the behaviours observed (50 (SD 10)).

(3) Macarthur-Bates Communicative Development Inventory (Words and Gestures) (MCDI) ${ }^{(43)}$ is a standardised parent reporting system used to assess monolingual children's lexical/vocabulary growth. The infant scale looks at comprehension, word production and aspects of symbolic and communicative gesture. Percentile ranks are computed from age (months) and sex-specific norms.

\section{Statistics}

Differences between the groups were determined by independent $t$ test where data were normally distributed, with comparisons being expressed as means and standard deviations. Where possible, non-parametric data were natural log-transformed to achieve a normal distribution as confirmed by the Kolmogorov-Smirnov test and probability plots. If data could not be normalised in this way, non-parametric MannWhitney $U$ tests were used. Correlative studies using Pearson's or Spearman's correlations (depending on data normality) were used to assess relationships between neurodevelopmental assessment results and individual FA proportions in cord blood and infant blood samples (in the combined study population). Linear regression analysis was used to determine significant predictors of neurodevelopmental test scores at the 18-month clinical visit, controlling for relevant covariates. 
All statistical analyses were performed using SPSS software (version 16 for PC). A $P$ value of $<0.05$ was considered statistically significant for all FA analyses.

\section{Results \\ Study participation}

The trial design and number of individuals at each stage are shown in Fig. 1. A total of 420 infants were randomised. In total, sixty-six infants withdrew following randomisation; forty-one from the treatment group and twenty-five from the placebo group. An additional number of participants could not be contacted to attend a follow-up visit, despite approximately three attempts via phone or letter. These participants were described as 'Did not attend visit' and are documented in Fig. 1 together with reasons for withdrawal. More participants in the treatment group withdrew compared with the placebo group, with FO associated with more withdrawals as a result of the smell. Overall, 287 were assessed for neurodevelopment, 149 from the placebo group and 138 from the FO group.

\section{Adherence and blinding}

The parents and the investigators remained blinded to the intervention until all the assessments were completed. Before unblinding, the parents were asked to indicate which supplement they believed they had been allocated to. Parents tended to base their assumption on the smell of the capsules, despite the use of peppermint flavouring in both oils; $92.2 \%$ of participants in the FO group correctly guessed their allocation, compared with $56 \cdot 25 \%$ of the placebo group. As aforementioned, the adherence to the supplementation was based upon a capsule count of the returned capsules and a daily diary kept by the participants. There was a statistically significant difference between the treatment groups for adherence $(t(322)=2 \cdot 129, P=0.034)$, with the FO group having significantly lower percentage adherence (55.3 (SD 33.2)) compared with the placebo group (62.6 (SD 28.4)). This was therefore assessed as a confounding factor in the subsequent analysis.

\section{Population characteristics}

There were no significant differences in the characteristics of the groups for any of the parameters examined including maternal age, parity, gestational length, neonatal anthropometric measures or incidence of allergic disease (Table 1). The population tended to be predominantly Caucasian, of high income, with high levels of both paternal and maternal education.

Due to the reduced number of participants who completed the neurodevelopment assessments relative to the total number of study participants, a comparison of the population characteristics of both groups was completed. For those participants who completed the BSID-III and CBCL, the Apgar score at 5 min was significantly higher (9.17 (SD 0.52)) when compared with those who did not attend (8.99 (SD 1.0 ); $P=0.026$ ), and both the maternal (attended: 33.5 (SD 4.3); did not attend: 31.5 (SD 4.8); $P=0.001$ ) and paternal (attended: 35.9 (SD 4.9), did not attend: 34.2 (SD 5.6); $P=0.002)$ ages were similarly higher for those participants who attended. For those who completed the MCDI (either 12 month or 18 month), only maternal age was significantly higher for those who completed the visit (attended: 33.6 (sD 4.3); did not attend: 32.3 (sD 4.6); $P=0.004$ ).

While the parental age of the participants who completed the assessment was increased, the income level, the number of siblings, the parental education level, if any other language are spoken at home, and the sex of the participants remained unchanged. Therefore, we can be reasonably confident that the group who completed the assessments is not likely to significantly differ from those who did not attend, and ensure the external validity of the study.

\section{Fatty acid measurements}

The erythrocyte FA measured at the end of the 6-month supplementation period showed significantly higher DHA $(P=0.03)$ and EPA $(P=0.016)$ levels and significantly lower AA levels $(P=0.003)$ in the FO group compared with the placebo group (Table 2). The plasma phospholipid FA showed significantly higher DHA $(P=0.001)$ and EPA levels $(P<0 \cdot 001)$, but AA levels remained unaltered $(P=0.376)$. Such results demonstrate that the supplementation was effective in significantly altering FA of the FO group. The levels of oleic acid in erythrocytes $(P=0.636)$ were not significantly different between the treatment groups; however, contrary to expectations, oleic acid levels in plasma phospholipids were significantly higher in the placebo group $(P=0 \cdot 012)$.

The erythrocyte and plasma phospholipid DHA values of the placebo group (5.78 (SD 1.5); 4.49 (SD 1.3), respectively) were comparable with other Australian breast-fed infants ${ }^{(42,46)}$. AA levels in plasma phospholipids and erythrocytes within both the placebo and FO groups remained within the range of values comparable with other Australian breast-fed infants ${ }^{(42,46)}$.

Within the FO-supplemented group, there was a significant correlation between the plasma phospholipid DHA levels at 6 months of age and the adherence to supplementation as measured by the diary/capsule count $\left(r_{\mathrm{s}}=0.426, P=0.001\right)$; however, a significant correlation was not observed for EPA or AA. For erythrocyte measurements, the opposite finding was observed, with AA and EPA significantly negatively correlated with adherence $\left(r_{\mathrm{s}}=-0.248, \quad P=0.05 ; \quad r_{\mathrm{s}}=0.373\right.$, $P=0.003$, respectively), and no significant correlation observed for DHA.

We also assessed DHA at birth to confirm that both groups had similar baseline levels before the supplement. There were no significant differences between the groups for the FA measurements taken from cord blood or breast milk (see Table 2). Cord blood DHA and AA levels were comparable with other populations within Western Australia ${ }^{(47)}$. 


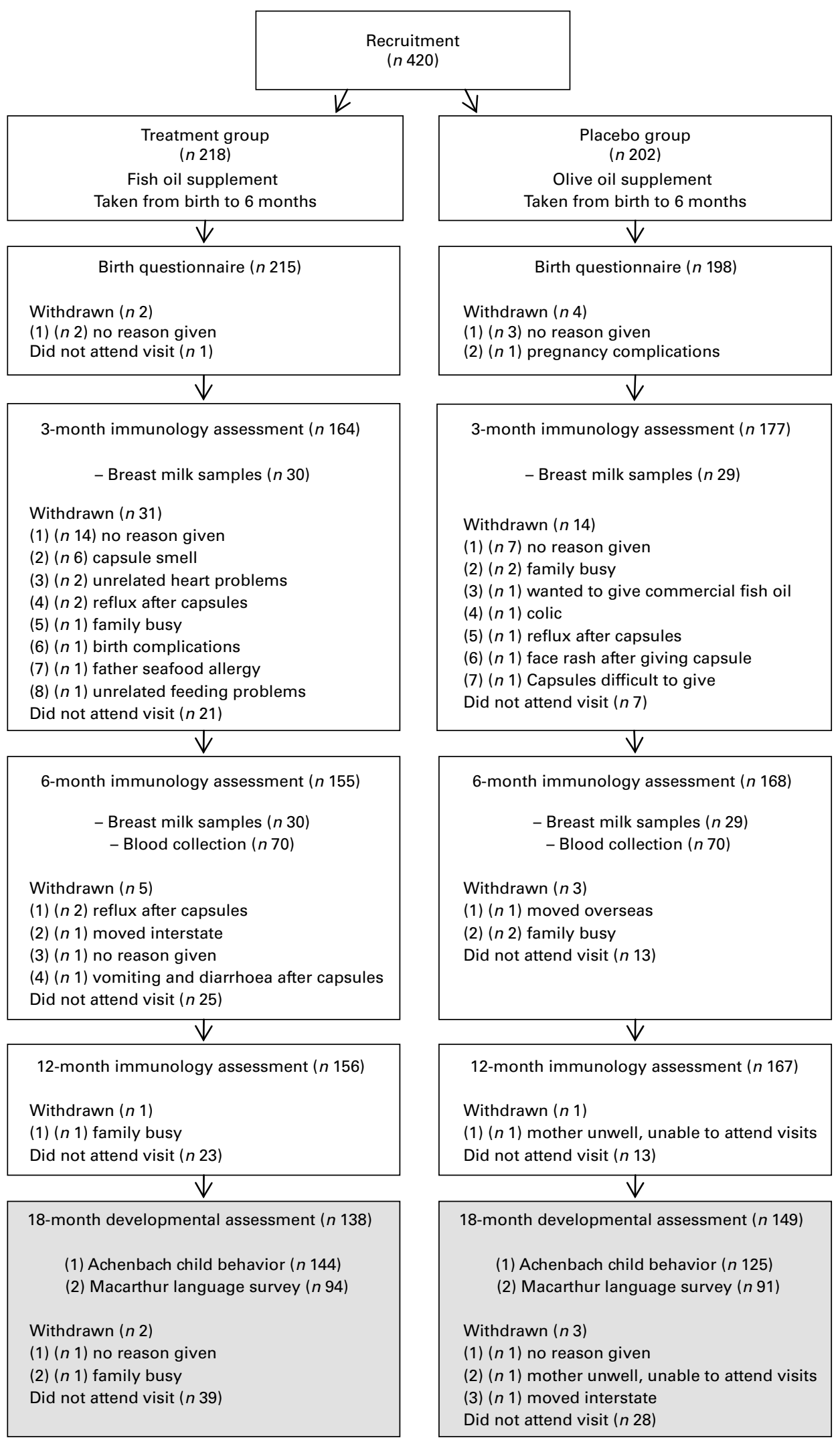

Fig. 1. Flow chart for study design, participant progress and data collection. 
Table 1. Population characteristics of the infants in the study population (Percentages, mean values and standard deviations)

\begin{tabular}{|c|c|c|c|c|}
\hline \multirow[b]{2}{*}{ Parameter } & \multicolumn{2}{|c|}{ Fish oil ( $n$ 138) } & \multicolumn{2}{|c|}{ Placebo ( $n$ 149) } \\
\hline & Mean & SD & Mean & SD \\
\hline \multicolumn{5}{|l|}{ Birth characteristics } \\
\hline Birth weight $(\mathrm{g})$ & 3443.61 & 409.4 & 3530.09 & 433.3 \\
\hline Birth length (cm) & $50 \cdot 16$ & $2 \cdot 7$ & $50 \cdot 63$ & $2 \cdot 3$ \\
\hline Sex (\% male) & \multicolumn{2}{|c|}{$51 \cdot 6$} & \multicolumn{2}{|c|}{51.5} \\
\hline Gestation (weeks) & $39 \cdot 1$ & $1 \cdot 1$ & 39.4 & 1.3 \\
\hline Apgar (5 min) & $9 \cdot 11$ & 0.82 & $9 \cdot 11$ & 0.56 \\
\hline Birth order (\% first born) & \multicolumn{2}{|c|}{48.5} & \multicolumn{2}{|c|}{$47 \cdot 0$} \\
\hline \multicolumn{5}{|l|}{ Family characteristics } \\
\hline Maternal education (years) & $15 \cdot 03$ & 2.5 & 14.79 & $2 \cdot 8$ \\
\hline Paternal education (years) & $14 \cdot 18$ & $2 \cdot 9$ & 14.47 & $2 \cdot 9$ \\
\hline Passive smoke exposure (\% not exposed) & \multicolumn{2}{|c|}{$51 \cdot 1$} & \multicolumn{2}{|c|}{$50 \cdot 3$} \\
\hline Ethnicity (\% Caucasian) & \multicolumn{2}{|c|}{$90 \cdot 1$} & \multicolumn{2}{|c|}{92.5} \\
\hline Only English spoken at home (\%) & \multirow{2}{*}{\multicolumn{2}{|c|}{$83 \cdot 9$}} & \multicolumn{2}{|c|}{89.6} \\
\hline \multicolumn{3}{|l|}{ Infant characteristics } & & \\
\hline 12-month height $(\mathrm{cm})$ & $75 \cdot 27$ & 8.4 & $76 \cdot 66$ & 3.5 \\
\hline 12 -month weight $(\mathrm{kg})$ & 11.79 & $12 \cdot 0$ & $11 \cdot 31$ & $10 \cdot 4$ \\
\hline 12-month head circumference $(\mathrm{cm})$ & $46 \cdot 9$ & $3 \cdot 7$ & $46 \cdot 8$ & $3 \cdot 7$ \\
\hline 12-month BMI $\left(\mathrm{kg} / \mathrm{m}^{2}\right)$ & 17.45 & 1.4 & $17 \cdot 28$ & 1.6 \\
\hline Allergic disease (\% diagnosed) & \multicolumn{2}{|c|}{$37 \cdot 8$} & \multicolumn{2}{|c|}{$39 \cdot 3$} \\
\hline Ever breast-fed (\%) & \multicolumn{2}{|c|}{97.5} & \multicolumn{2}{|c|}{$98 \cdot 2$} \\
\hline Age ceased breast-feeding (months) & $6 \cdot 67$ & 3.5 & $6 \cdot 37$ & $3 \cdot 6$ \\
\hline Breast-fed $>6$ months (\%) & \multicolumn{2}{|c|}{44.6} & \multicolumn{2}{|c|}{53.1} \\
\hline Ever formula-fed (\%) & \multirow{2}{*}{\multicolumn{2}{|c|}{$\begin{array}{l}73 \cdot 9 \\
57 \cdot 1\end{array}$}} & \multirow{2}{*}{\multicolumn{2}{|c|}{$\begin{array}{c}82 \cdot 4 \\
50\end{array}$}} \\
\hline Given LC-PUFA formula & & & & \\
\hline Age commenced solids (months) & $5 \cdot 63$ & 0.98 & 5.51 & 0.78 \\
\hline BSID-III assessment age (months; d) & \multicolumn{2}{|c|}{$18 ; 16$} & \multicolumn{2}{|c|}{$18 ; 03$} \\
\hline MCDI 12-month age (months; d) & \multirow{2}{*}{\multicolumn{2}{|c|}{$12 ; 01$}} & \multicolumn{2}{|c|}{$11 ; 09$} \\
\hline MCDI and CBCL 18-month age (months; d) & & $18 ; 00$ & \multicolumn{2}{|c|}{$18 ; 00$} \\
\hline
\end{tabular}

LC-PUFA, long chain PUFA; BSID-III, Bayley Scales of Infant and Toddler Development (3rd edition); MCDI, Macarthur-Bates Communicative Development Inventory; CBCL, Child Behavior Checklist.

The breast milk levels of DHA in the study population $(0 \cdot 34$ (SD 15)\%) were higher than previously observed levels within Australia of $0 \cdot 20 \%$ total $\mathrm{FA}^{(35)}$. This may be due to the high socio-economic status of the participants and awareness of the potential benefits of high $\mathrm{FO} /$ fish intake during lactation as a result of study participation. Levels of AA, however, were comparable with previous data ${ }^{(35)}$. No EPA could be detected in the breast milk at either time point.

Table 2. Fatty acid measurements (\% total fatty acids) taken during the course of the trial for the fish oil and placebo groups (Mean values and standard deviations)

\begin{tabular}{|c|c|c|c|c|c|c|c|c|c|c|c|c|c|}
\hline \multirow[b]{2}{*}{ Fatty acid } & \multirow[b]{2}{*}{ Group } & \multicolumn{2}{|c|}{$\begin{array}{c}\text { Maternal FFQ } \\
(\mathrm{g} / \mathrm{d})(n \text { 375) }\end{array}$} & \multicolumn{2}{|c|}{$\begin{array}{l}\text { Cord blood } \\
\text { erythrocyte } \\
\quad(n 109)\end{array}$} & \multicolumn{2}{|c|}{$\begin{array}{l}3 \text { Months breast } \\
\text { milk }(n 64)\end{array}$} & \multicolumn{2}{|c|}{$\begin{array}{l}6 \text { Months breast } \\
\text { milk ( } n 59)\end{array}$} & \multicolumn{2}{|c|}{$\begin{array}{l}6 \text { Months } \\
\text { erythrocyte } \\
(n 139)\end{array}$} & \multicolumn{2}{|c|}{$\begin{array}{c}6 \text { Months } \\
\text { plasma }(n 145)\end{array}$} \\
\hline & & Mean & SD & Mean & SD & Mean & SD & Mean & SD & Mean & SD & Mean & SD \\
\hline \multirow[t]{2}{*}{$18: 1 n-9$ OA } & Fish oil & \multicolumn{2}{|c|}{ NM } & $13 \cdot 29$ & $6 \cdot 2$ & $37 \cdot 24$ & 3.5 & 35.47 & $2 \cdot 7$ & $16 \cdot 49$ & $4 \cdot 1$ & $10 \cdot 15^{\star}$ & 1.6 \\
\hline & Olive oil & \multicolumn{2}{|c|}{ NM } & $13 \cdot 71$ & $5 \cdot 2$ & 36.46 & 3.4 & 36.44 & 3.5 & $16 \cdot 83$ & 4.5 & $10 \cdot 89^{*}$ & $1 \cdot 8$ \\
\hline \multirow[t]{2}{*}{$18: 2 n-6$ LA } & Fish oil & 10.59 & $4 \cdot 8$ & $3 \cdot 71$ & 0.51 & $10 \cdot 29$ & $2 \cdot 1$ & $10 \cdot 26$ & $2 \cdot 3$ & 9.29 & $1 \cdot 7$ & 19.59 & 3.0 \\
\hline & Olive oil & 9.90 & 4.7 & 3.81 & 0.92 & $10 \cdot 43$ & $2 \cdot 6$ & $9 \cdot 75$ & 1.9 & 9.59 & $1 \cdot 6$ & $20 \cdot 41$ & 3.2 \\
\hline \multirow[t]{2}{*}{$18: 3 n-3$ ALA } & Fish oil & 0.87 & 0.37 & 0.496 & $0 \cdot 13$ & $1 \cdot 1$ & 0.35 & 1.02 & 0.34 & 0.476 & 0.11 & 0.110 & 0.03 \\
\hline & Olive oil & 0.89 & 0.49 & 0.513 & 0.15 & 0.99 & 0.29 & 1.01 & 0.38 & 0.481 & 0.09 & 0.114 & 0.04 \\
\hline \multirow[t]{2}{*}{$20: 3 n-6$ DGLA } & Fish oil & \multicolumn{2}{|c|}{ NM } & 2.98 & 0.77 & 0.35 & 0.07 & 0.296 & 0.07 & 1.35 & 0.37 & $2 \cdot 27$ & 0.49 \\
\hline & Olive oil & \multicolumn{2}{|c|}{ NM } & $3 \cdot 10$ & $0 \cdot 10$ & 0.32 & 0.09 & 0.267 & 0.06 & 1.36 & 0.35 & $2 \cdot 32$ & 0.59 \\
\hline \multirow[t]{2}{*}{$20: 4 n-6$ AA } & Fish oil & 0.87 & 0.37 & $15 \cdot 76$ & $1 \cdot 77$ & 0.40 & 0.07 & 0.38 & 0.07 & $14 \cdot 59^{\star \star}$ & $1 \cdot 7$ & $10 \cdot 65$ & 1.9 \\
\hline & Olive oil & 0.84 & 0.49 & $15 \cdot 54$ & $2 \cdot 15$ & 0.39 & $0 \cdot 10$ & 0.36 & 0.1 & $16 \cdot 55^{\star \star}$ & $2 \cdot 3$ & 10.94 & 1.9 \\
\hline \multirow[t]{2}{*}{$20: 5 n-3$ EPA } & Fish oil & 0.07 & 0.05 & 0.334 & 0.14 & 0.015 & 0.06 & \multicolumn{2}{|c|}{ Trace } & $0 \cdot 917^{*}$ & 0.42 & $1 \cdot 2^{\star *}$ & 0.56 \\
\hline & Olive oil & 0.06 & 0.04 & 0.308 & 0.09 & 0.00 & 0.00 & \multirow{2}{*}{\multicolumn{2}{|c|}{ Trace }} & $0 \cdot 761^{*}$ & 0.42 & $0.89^{* *}$ & 0.37 \\
\hline \multirow[t]{2}{*}{$22: 5 n-3$ DPA } & Fish oil & \multicolumn{2}{|c|}{ NM } & 0.700 & 0.22 & \multicolumn{2}{|c|}{ NM } & \multirow{2}{*}{\multicolumn{2}{|c|}{$\begin{array}{l}\text { NM } \\
\text { NM }\end{array}$}} & 1.92 & 0.49 & 0.98 & 0.32 \\
\hline & Olive oil & & & 0.673 & $0 \cdot 17$ & $\mathrm{~N}$ & & & & $1 \cdot 82$ & 0.58 & 0.88 & 0.38 \\
\hline \multirow[t]{2}{*}{$22: 6 n-3 \mathrm{DHA}$} & Fish oil & 0.09 & 0.08 & $7 \cdot 36$ & $1 \cdot 15$ & 0.35 & $0 \cdot 17$ & 0.35 & 0.23 & $6 \cdot 83^{\star}$ & $1 \cdot 7$ & $5 \cdot 34^{\star *}$ & $1 \cdot 6$ \\
\hline & Olive oil & 0.08 & 0.08 & 7.44 & 1.44 & 0.32 & $0 \cdot 13$ & 0.34 & 0.22 & $6 \cdot 22^{*}$ & $1 \cdot 6$ & $4 \cdot 49^{\star *}$ & $1 \cdot 3$ \\
\hline
\end{tabular}

OA, oleic acid; NM, not measured; LA, linoleic acid; ALA, $\alpha$-linolenic acid; DGLA, dihomo- $\gamma$-linolenic acid; AA, arachidonic acid; DPA, docosapentaenoic acid Mean values were significantly different between the fish oil and placebo groups: * $P<0.05,{ }^{\star \star} P<0.01$

${ }^{*}$ Percentage of total fatty acids. 


\section{Bayley Scales of Infant and Toddler Development}

There were no significant differences between the groups for the standard or composite scores in any of the BSID-III subtests. As shown in Table 3, the mean scores for each subtest tended to be higher for the FO group, but this did not reach statistical significance. Of interest to note is the tendency for low scores on the MCDI for this population. One reason for this may be that this is an American assessment, and some of the vocabulary is not appropriate to an Australian population, and this is reflected in the scores being lower when compared with the normative US sample.

\section{Child Behavior Checklist}

As expected for a typically developing term infant population, the mean scores of both supplementation groups were heavily skewed towards the $T$-score of 50 , the lowest score achievable, and normality could not be obtained statistically. As a result, the data were analysed categorically, with the percentages of children who obtained a $T$-score $>50$ comparable between the groups. As shown in Table 4, there was a significant difference between the groups for anxious/depressed behaviours, with more children in the FO group scoring $>50$ $T$-score relative to the placebo group $\left(\chi^{2}(1, n 265)=5.580\right.$, $P=0 \cdot 018)$. This remained significant after adjusting for capsule compliance $(\beta=0.5,95 \%$ CI $1 \cdot 014,2 \cdot 838, P=0.044)$. As shown in Tables 4 and 5 , there were no significant differences between the groups in any of the remaining CBCL subscales. There were also no differences in the number of participants in the borderline or clinical range for the CBCL subtests between the study groups (data not shown), either before or after adjusting for capsule compliance.

Table 3. Bayley Scales of Infant and Toddler Development (3rd edition; BSID-III) outcomes; fish oil compared with placebo at 18 months of age (Mean values and standard deviations)

\begin{tabular}{|c|c|c|c|c|c|}
\hline \multirow[b]{2}{*}{ Outcome } & \multicolumn{2}{|c|}{$\begin{array}{l}\text { Fish oil } \\
(n 138)\end{array}$} & \multicolumn{2}{|c|}{$\begin{array}{l}\text { Placebo } \\
(n 149)\end{array}$} & \multirow[b]{2}{*}{$P$} \\
\hline & Mean & SD & Mean & SD & \\
\hline \multicolumn{6}{|l|}{$\begin{array}{l}\text { BSID standard } \\
\text { scores }\end{array}$} \\
\hline Cognitive & 11.55 & $2 \cdot 2$ & 11.43 & $2 \cdot 3$ & 0.526 \\
\hline $\begin{array}{l}\text { Receptive } \\
\text { language }\end{array}$ & 12.02 & 2.5 & 11.66 & 2.9 & 0.256 \\
\hline $\begin{array}{l}\text { Expressive } \\
\text { language }\end{array}$ & 9.94 & $3 \cdot 1$ & 9.52 & $2 \cdot 7$ & 0.232 \\
\hline Fine motor & 11.86 & 1.7 & 11.84 & 3.7 & 0.957 \\
\hline Gross motor & $9 \cdot 81$ & $1 \cdot 7$ & 9.77 & 1.8 & 0.867 \\
\hline $\begin{array}{l}\text { Social } \\
\text { emotional }\end{array}$ & $12 \cdot 37$ & 3.9 & $12 \cdot 02$ & 4.0 & 0.538 \\
\hline \multicolumn{6}{|l|}{$\begin{array}{l}\text { BSID composite } \\
\text { scores }\end{array}$} \\
\hline Cognitive & 107.65 & 11.6 & $105 \cdot 28$ & 19.9 & 0.226 \\
\hline Language & $104 \cdot 71$ & $13 \cdot 7$ & 104.07 & $16 \cdot 3$ & 0.722 \\
\hline Motor & 105.42 & $8 \cdot 8$ & 103.43 & $11 \cdot 1$ & 0.097 \\
\hline $\begin{array}{l}\text { Social } \\
\quad \text { emotional }\end{array}$ & 111.6 & 19.5 & $110 \cdot 42$ & $19 \cdot 9$ & 0.674 \\
\hline $\begin{array}{l}\text { Adaptive } \\
\text { behaviour }\end{array}$ & 96.98 & $12 \cdot 4$ & 97.84 & 13.8 & 0.648 \\
\hline
\end{tabular}

Table 4. Categorical Child Behavior Checklist outcomes; fish oil compared with placebo at 18 months of age

\begin{tabular}{|c|c|c|c|}
\hline \multirow[b]{2}{*}{ Outcome } & \multicolumn{3}{|c|}{$\begin{array}{l}\text { Percentage of participants with } \\
\text { a } T \text {-score }>50\end{array}$} \\
\hline & $\begin{array}{l}\text { Fish oil } \\
(n 125)\end{array}$ & $\begin{array}{l}\text { Placebo } \\
(n 144)\end{array}$ & $P$ \\
\hline \multicolumn{4}{|l|}{ Empirically based scores } \\
\hline Emotionally reactive & $35 \cdot 2$ & $41 \cdot 1$ & 0.320 \\
\hline Anxious/depressed & $47 \cdot 6$ & 33.4 & $0.018^{*}$ \\
\hline Somatic complaints & 18.5 & $22 \cdot 7$ & 0.406 \\
\hline Withdrawn & $42 \cdot 6$ & $37 \cdot 9$ & 0.441 \\
\hline Sleep problems & 43.5 & 39.0 & 0.453 \\
\hline Attention problems & 64.6 & $60 \cdot 9$ & 0.554 \\
\hline Aggressive behaviour & $45 \cdot 4$ & $50 \cdot 0$ & 0.453 \\
\hline \multicolumn{4}{|l|}{ DSM-oriented scores } \\
\hline Affective problems & 58.9 & $65 \cdot 3$ & 0.280 \\
\hline Anxiety problems & 31.2 & 29.8 & 0.810 \\
\hline $\begin{array}{l}\text { Pervasive developmental } \\
\text { problems }\end{array}$ & $43 \cdot 5$ & $47 \cdot 5$ & 0.517 \\
\hline $\begin{array}{l}\text { Attention deficit/hyperactivity } \\
\text { problems }\end{array}$ & $80 \cdot 6$ & $77 \cdot 3$ & 0.506 \\
\hline $\begin{array}{l}\text { Oppositional defiance } \\
\text { problems }\end{array}$ & $52 \cdot 8$ & $48 \cdot 9$ & 0.526 \\
\hline
\end{tabular}

DSM, Diagnostic and Statistical Manual of Mental Disorders. ${ }^{\star} P<0.05$.

\section{Macarthur-Bates Communicative Development Inventory}

Overall, a lower number of participants were able to complete the MCDI, as the use of this questionnaire was not introduced until October 2007, and therefore the first 224 participants did not complete the 12-month questionnaire, and the first 164 participants did not complete the 18-month questionnaire.

As illustrated in Table 6 , children in the FO group performed significantly better in language assessments at 12 and 18 months of age with higher percentile ranks of both later developing gestures $(P=0.007 ; P=0.002$, respectively) and the total number of gestures $(P=0.023 ; P=0.006$, respectively) compared with placebo. These relationships remained significant after adjusting for potential confounding effects of capsule compliance (later developing gestures at 12 months: $\beta=0 \cdot 247,95 \%$ CI $3 \cdot 433,24 \cdot 214, P=0 \cdot 010$; later developing gestures at 18 months: $\beta=0 \cdot 224$, 95\% CI 3.604 , $22 \cdot 670, P=0.007$; total number of gestures at 12 months: $\beta=0 \cdot 238,95 \%$ CI $2 \cdot 846,23 \cdot 642, P=0.013$, total number of gestures at 18 months: $\beta=0 \cdot 211,95 \%$ CI $2 \cdot 685,21 \cdot 103$, $P=0.012$ ). No other significant differences were observed for the remainder of the data collected using the MCDI. While the authors of the MCDI advise that for young infants that small differences in raw scores can produce large shifts in percentile scores ${ }^{(43)}$, in the present analysis, raw scores were also evaluated statistically, and the same significant differences for the gestural subtests were observed (data not shown). The change between raw scores $(\delta)$ at 12 months and 18 months for each subscore of the MCDI was evaluated statistically to evaluate whether the supplementation had any effect upon the rate of language acquisition. No significant effect of group was observed for the $\delta$ for any of the MCDI subscores (data not shown). 
Table 5. Linear Child Behavior Checklist outcomes; fish oil compared with placebo at 18 months of age

(Mean values and standard deviations)

\begin{tabular}{|c|c|c|c|c|c|}
\hline \multirow[b]{2}{*}{ Outcome } & \multicolumn{2}{|c|}{$\begin{array}{l}\text { Fish oil } \\
(n 125)\end{array}$} & \multicolumn{2}{|c|}{$\begin{array}{c}\text { Placebo } \\
(n 144)\end{array}$} & \multirow[b]{2}{*}{$P$} \\
\hline & Mean & SD & Mean & SD & \\
\hline Internalising behaviours & 41.77 & $8 \cdot 4$ & 41.99 & $8 \cdot 4$ & 0.832 \\
\hline Externalising behaviours & $46 \cdot 90$ & $8 \cdot 2$ & $47 \cdot 18$ & 8.7 & 0.735 \\
\hline Total behaviours & 44.73 & $7 \cdot 6$ & 44.95 & $8 \cdot 2$ & 0.824 \\
\hline
\end{tabular}

\section{Fatty acid values and neurodevelopmental outcomes}

Infant erythrocyte DHA measurements at 6 months of age significantly predicted communication skills in the Adaptive Behaviour Questionnaire of the BSID-III ( $\beta=0 \cdot 291$, 95\% CI $0 \cdot 115,0 \cdot 870, P=0 \cdot 011)$ and remained significant after controlling for allergic disease, parity, maternal education, capsule consumption and sex $(\beta=0.281,95 \%$ CI $0.043,0.979$, $P=0 \cdot 007)$. No other significant associations were observed between infant erythrocyte DHA and neurodevelopmental outcomes.

Infant plasma phospholipid measurements at 6 months of age significantly predicted the phrases understood scores in the MCDI questionnaire at 12 months of age $(\beta=0.468$, $95 \%$ CI $3.726,12 \cdot 136, P<0.001$ ), and remained a trend, but did not remain statistically significant after controlling for allergic disease, capsule consumption, parity, maternal education and $\operatorname{sex}(\beta=0 \cdot 310,95 \%$ CI $-0 \cdot 080,10 \cdot 588, P=0.053)$.

When we examined the FA at birth, we found no significant associations between cord blood DHA measurements and neurodevelopmental outcomes.

\section{Discussion}

The present study is the first randomised controlled trial evaluating the effects of direct high-dose FO supplementation in term infants on neurodevelopment including language acquisition. The findings do not fully support our hypothesis, with no observed effects of FO supplementation on neurodevelopmental skills as assessed by the Bayley Scales. However, in a smaller subset analysis, supplementation resulted in higher gesture scores on the MCDI compared with placebo, both at 12 and 18 months of age. As gesture precedes spoken work acquisition and is associated with later developing vocabulary skills ${ }^{(48)}$, such data study presents a case for future larger multi-centre studies to confirm this effect and address

Table 6. Macarthur-Bates Communicative Development Inventory raw scores and percentile ranks; fish oil compared with placebo at 12 and 18 months of age

(Number of participants, mean values and standard deviations)

\begin{tabular}{|c|c|c|c|c|c|}
\hline \multirow[b]{2}{*}{ Outcome } & \multicolumn{2}{|c|}{ Fish oil } & \multicolumn{2}{|c|}{ Placebo } & \multirow[b]{2}{*}{$P$} \\
\hline & Mean & SD & Mean & SD & \\
\hline \multicolumn{6}{|l|}{ 12-month raw scores } \\
\hline$n$ & \multicolumn{2}{|c|}{62} & \multicolumn{2}{|c|}{66} & \\
\hline Phrases understood & $13 \cdot 34$ & 6.7 & $13 \cdot 60$ & $5 \cdot 8$ & 0.868 \\
\hline Words understood & $68 \cdot 30$ & $47 \cdot 6$ & 61.42 & $52 \cdot 2$ & 0.488 \\
\hline Words spoken & $6 \cdot 11$ & $7 \cdot 5$ & $5 \cdot 52$ & $8 \cdot 7$ & 0.276 \\
\hline Early gestures & $10 \cdot 29$ & 3.5 & $9 \cdot 56$ & $3 \cdot 14$ & 0.152 \\
\hline Later gestures & $15 \cdot 16$ & $8 \cdot 3$ & $11 \cdot 26$ & $7 \cdot 5$ & $0.017^{\star}$ \\
\hline Total gestures & $25 \cdot 47$ & $10 \cdot 9$ & $20 \cdot 76$ & $10 \cdot 1$ & $0.026^{\star}$ \\
\hline \multicolumn{6}{|l|}{ 12-month percentile rank } \\
\hline$n$ & \multicolumn{2}{|c|}{62} & \multicolumn{2}{|c|}{66} & \\
\hline Phrases understood & $46 \cdot 12$ & $26 \cdot 1$ & $46 \cdot 80$ & 23.4 & 0.875 \\
\hline Words understood & $35 \cdot 79$ & $26 \cdot 2$ & $34 \cdot 71$ & $27 \cdot 8$ & 0.314 \\
\hline Words spoken & 41.57 & $35 \cdot 8$ & $37 \cdot 04$ & $25 \cdot 5$ & 0.537 \\
\hline Early gestures & $51 \cdot 18$ & $27 \cdot 9$ & $45 \cdot 81$ & $27 \cdot 9$ & 0.275 \\
\hline Later gestures & $55 \cdot 21$ & $26 \cdot 4$ & $42 \cdot 12$ & 27.9 & $0.007^{\star *}$ \\
\hline Total gestures & $50 \cdot 50$ & $27 \cdot 7$ & $38 \cdot 81$ & $27 \cdot 7$ & $0.012^{*}$ \\
\hline \multicolumn{6}{|l|}{ 18-month raw scores } \\
\hline$n$ & \multicolumn{2}{|c|}{77} & \multicolumn{2}{|c|}{84} & \\
\hline Phrases understood & $24 \cdot 06$ & $4 \cdot 7$ & $23 \cdot 50$ & $5 \cdot 1$ & 0.593 \\
\hline Words understood & 199.09 & $83 \cdot 7$ & $190 \cdot 43$ & 94.5 & 0.515 \\
\hline Words spoken & $49 \cdot 16$ & $55 \cdot 8$ & $58 \cdot 50$ & 63.5 & 0.486 \\
\hline Early gestures & 14.09 & $2 \cdot 3$ & $13 \cdot 62$ & $7 \cdot 7$ & $0 \cdot 240$ \\
\hline Later gestures & $30 \cdot 81$ & $7 \cdot 6$ & $28 \cdot 08$ & $7 \cdot 7$ & $0.049^{\star}$ \\
\hline Total gestures & $44 \cdot 75$ & $9 \cdot 0$ & $41 \cdot 48$ & $9 \cdot 3$ & $0.043^{\star}$ \\
\hline \multicolumn{6}{|l|}{ 18-month percentile rank } \\
\hline$n$ & \multicolumn{2}{|c|}{77} & \multicolumn{2}{|c|}{84} & \\
\hline Phrases understood & $60 \cdot 91$ & $33 \cdot 8$ & $56 \cdot 89$ & 33.6 & 0.445 \\
\hline Words understood & $35 \cdot 79$ & $28 \cdot 2$ & $34 \cdot 71$ & $27 \cdot 7$ & 0.804 \\
\hline Words spoken & $27 \cdot 22$ & $24 \cdot 5$ & $27 \cdot 80$ & $27 \cdot 8$ & 0.392 \\
\hline Early gestures & $27 \cdot 79$ & $24 \cdot 1$ & $22 \cdot 51$ & 23.7 & 0.158 \\
\hline Later gestures & 43.59 & $29 \cdot 7$ & 29.99 & $28 \cdot 8$ & $0.003^{\star \star}$ \\
\hline Total gestures & $42 \cdot 22$ & $28 \cdot 5$ & $30 \cdot 77$ & $27 \cdot 7$ & $0.010^{\star *}$ \\
\hline
\end{tabular}

${ }^{\star} P<0.05,{ }^{\star *} P<0.01$. 
longer-term outcomes of language development. Additionally, the increased score in the anxiety/depression subscale of the CBCL in the present trial does not support a beneficial effect of early FO supplementation on mood.

There have been several previous trials of LC-PUFA supplementation in term infants that have investigated language outcomes, yet results from these trials have not demonstrated consistent benefits to language development ${ }^{(14,16,17,31,49)}$. Similarly, there is no existing evidence suggesting that enhanced n-3 LC-PUFA status or supplementation with $n-3$ LC-PUFA is likely to functionally manifest in negative effects upon mental health development. Indeed, in adults, FO supplementation has been noted as protective for mental health $^{(50,51)}$. The present findings are also in contrast to the most recent Cochrane review ${ }^{(33)}$, which concluded that the majority of well-designed previous trials of LC-PUFA supplementation of infant formula have not shown effects upon neurodevelopment. A number of differences between the present trial and previous studies may have contributed to the observed benefit of postnatal FO supplementation.

First, the method used for the assessment of neurodevelopment could be an important factor. In comparison with global standardised measures of infant development such as the BSID-III, detailed parent-report measures of development such as the MCDI or CBCL represent a cost-effective and highly valid instrument for which to assess a specific facet of cognitive development. Direct assessments evaluate infant skills in a decontextualised environment, which can fail to capture their true functional capacities ${ }^{(52)}$. Additionally, the BSID-III is designed to diagnose a delay/disorder in neurodevelopment, which may not be appropriate in determining the potentially subtle effects of a nutritional supplement on normally developing, healthy term infants ${ }^{(53)}$.

Second, the LC-PUFA levels measured at the end of the supplementation period indicated that the increase in DHA status within the treatment group was statistically significant, supporting the hypothesis that supplementation with high-dose FO would significantly increase infant FA status. Furthermore, both infant erythrocyte and plasma DHA status at 6 months of age predicted scores of language/communications skills, lending further support to the group differences between $n-3$ LC-PUFA and communicative development observed. Finally, the study population is unique in its inclusion of all infants regardless of the feeding practice (i.e. breast or bottle-fed), which increases its wider applicability. Nevertheless, it remains possible that a number of alternative factors may have had an impact on the results observed. Such factors may limit confidence in the group differences observed.

Despite our best efforts to match the capsules, the majority $(92.9 \%)$ of participants in the FO group correctly guessed their infant's group allocation. The precise effects of this are unknown, but considering the wide commercialisation of FO capsules as promoting intellectual development, it is feasible that parents within the FO-supplemented group may have altered perceptions of their child's skills. This is unlikely to affect measures such as the BSID-III where the children were assessed by an examiner unaware of the group allocation, but it may have had an impact on parent-report measures such as the MCDI or CBCL.

The discrepant sample sizes between the assessment measures remain a potential for bias within the study. Although with approximately 140 participants per group, the present study did have adequate power to detect differences according to the BSID-III, the number of completed MCDI assessments was lower, at approximately 81 per group. However, this was not due to self-selection bias, as the MCDI was added to the assessment protocol at a later stage of the trial. Still, our sample population was biased to high-income, well-educated, predominantly Caucasian population, which may be expected to have good knowledge of healthy nutrition. This population is also less likely to be 'deficient' in DHA, as reflected by the comparatively high levels of DHA in breast milk.

The present trial was part of a large randomised controlled trial designed to assess the role of FO in allergy prevention ${ }^{(37)}$. Infants at high risk of developing allergic disease due to maternal atopy were recruited, as they were at a greater risk of atopy than parents with no family history ${ }^{(54)}$. Such a selected population limits the diversity of the study population. Although the exact implications of this population selection cannot be precisely determined, allergic disease has been associated with neurodevelopment in a bidirectional manner ${ }^{(55)}$, and this may limit the applicability of the present results for a population not predisposed to allergic disease. Further, despite the fact that randomisation should have ensured that confounding factors were balanced evenly between the groups, an environmental survey or questionnaire such as the HOME inventory at the time of developmental testing would have been beneficial to ascertaining whether any imbalance in the home environment occurred between the randomised groups.

With regard to the erythrocyte LC-PUFA levels, while supplementation successfully increased the DHA status of the treatment group, this increase may be interpreted as modest considering the dose. Gibson et al. ${ }^{(30)}$ showed that increasing breast milk levels to approximately $1.13 \%$ DHA resulted in infant erythrocyte phospholipid values of $9 \cdot 8 \%$ total FA. Considering that the dosage in the present study was approximately $1 \%$ FA, the mean infant erythrocyte DHA value of $6.5 \%$ appears to be lower than expected. One possible reason for this may be capsule adherence, as the FO group reported adherence at a mean of $59 \%$, thus the dosage is likely to be lower overall than anticipated. A second possible factor is that the majority of the population was already receiving a significant quantity of DHA from breast milk, as DHA levels on average were higher for this cohort than the typical Australian population $^{(35)}$. Other considerations include the method of delivery $^{(56)}$, which may have resulted in a variable amount of supplement adequately ingested, and the bioavailability of the ethyl ester supplement ${ }^{(57,58)}$, both of which may have reduced the effectiveness of the supplement in increasing n-3 LC-PUFA levels.

In contrast, the LC-PUFA levels measured in plasma phospholipids indicated that the increase in DHA status within the treatment group was more substantial. However, the 
statistically significant increase in oleic acid levels in the placebo group does cast doubt as to whether olive oil was an appropriate placebo, which was surprising, taking into account how trivial the amount of oleic acid provided in the supplement compares to the quantity within breast milk or infant formula ${ }^{(59)}$. Plasma phospholipid measurements may reflect short-term intake, therefore compliance may have increased near to the clinical visit in which the sample was taken, as has been observed in previous clinical trials ${ }^{(60)}$.

Several assessment measures were completed within the present trial in order to increase the likelihood of identifying a particular facet of neurodevelopment affected by $n$-3 LCPUFA supplementation. However, this increased the type 1 error rate due to the high number of statistical comparisons completed. Although multiple comparisons are inevitable in studies of this nature, the statistical corrections that are often employed to address this (e.g. Bonferroni correction) infer that multiple relationships (even if consistent and significant) detract from each other, and deal with this by adjustments that abolish any findings without extremely significant levels ( $P$ values). However, it has been validly argued $^{(61)}$ that where there are consistent, repeated, coherent and biologically plausible patterns, the results 'reinforce' rather than detract from each other (even if $P$ values are significant but not very large). We argue that this may be the case with the consistent relationships between $n$ - 3 LC-PUFA status and the multiple neurodevelopment measures observed in the present study.

In conclusion, the present findings suggest that FO supplementation during early infancy resulted in higher gestural scores, yet no benefit for overall development or behaviour measured at 18 months in healthy term children. Further, children within the FO group exhibited some evidence of increased anxious/depressed behaviours. However, to confirm such findings, further studies are required with larger sample sizes of a more diverse population. The present study has demonstrated that adherence was reduced using this method of direct supplementation, reinforcing that maternal supplementation during lactation or infant formula may be a more desirable way to achieve higher DHA status, and potentially more conclusive results of the overall effects of high $n-3$ LCPUFA supplementation during early infancy may be obtained.

\section{Acknowledgements}

This Infant Fish Oil Supplementation Study (IFOS) was funded by the National Health and Medical Research Council (NHMRC) of Australia (grant ID 458502). S. L. P. was supported by a NHMRC Practitioner Fellowship. S. J. M. was supported by a PhD Scholarship from the Women \& Infants' Research Foundation. N. D. was supported by a PhD Scholarship from the Asthma Foundation of Australia. We appreciate the contributions of Professor Trevor Mori, Mrs Suzi McCarthy, Dr Meri Tulic, Dr Ylennia Casadio, Dr Elaine Pascoe and Ms Glenys Dixon in addition to the entire Childhood Allergy \& Immunology Research Group staff. We also gratefully acknowledge the infants and their families for their participation in this study. The contributions of each author were as follows: S. J. M. was involved in the participant recruitment, data collection, statistical analysis, drafting and editing of the manuscript. S. L. P., J. A. D., K. H. and K. S. conceived and designed the study. N. D. was involved in participant recruitment and data collection. All authors edited the paper content and approved the final manuscript. There are no relevant disclosures pertaining to funding or conflict of interest issues.

\section{References}

1. Salem N Jr, Litman B, Kim HY, et al. (2001) Mechanisms of action of docosahexaenoic acid in the nervous system. Lipids 36, 945-959.

2. Innis SM (2007) Dietary ( $n-3)$ fatty acids and brain development. $J$ Nutr 137, 855-859.

3. Innis SM (2003) Perinatal biochemistry and physiology of long-chain polyunsaturated fatty acids. J Pediatr 143, S1-S8.

4. Hornstra G (2000) Essential fatty acids in mothers and their neonates. Am J Clin Nutr 71, 1262S-1269S.

5. Simopoulos AP (1991) Omega-3 fatty acids in health and disease and in growth and development. Am J Clin Nutr 54, 438-463.

6. Drover J, Hoffman DR, Castaneda YS, et al. (2009) Three randomized controlled trials of early long-chain polyunsaturated fatty acid supplementation on means-end problem solving in 9-month-olds. Child Dev 80, 1376-1384.

7. Willatts P, Forsyth JS, DiModugno MK, et al. (1998) Effect of long-chain polyunsaturated fatty acids in infant formula on problem solving at 10 months of age. Lancet 352, 688-691.

8. Willatts P, Forsyth JS, DiModugno MK, et al. (1998) Influence of long-chain polyunsaturated fatty acids on infant cognitive function. Lipids 33, 973-980.

9. Scott DT, Janowsky JS, Carroll RE, et al. (1998) Formula supplementation with long-chain polyunsaturated fatty acids: are there developmental benefits? Pediatrics 102, E59.

10. Makrides M, Neumann M, Simmer K, et al. (1995) Are longchain polyunsaturated fatty acids essential nutrients in infancy? Lancet 345, 1463-1468.

11. de Jong C, Kikkert HK, Fidler V, et al. (2010) The Groningen LCPUFA study: no effect of postnatal long-chain polyunsaturated fatty acids in healthy term infants on neurological condition at 9 years. Br J Nutr 104, 566-572.

12. Bouwstra H, Dijck-Brouwer DA, Wildeman JA, et al. (2003) Long-chain polyunsaturated fatty acids have a positive effect on the quality of general movements of healthy term infants. Am J Clin Nutr 78, 313-318.

13. Birch EE, Hoffman DR, Castaneda YS, et al. (2002) A randomized controlled trial of long-chain polyunsaturated fatty acid supplementation of formula in term infants after weaning at 6 wk of age. Am J Clin Nutr 75, 570-580.

14. Birch EE, Garfield S, Hoffman DR, et al. (2000) A randomized controlled trial of early dietary supply of long-chain polyunsaturated fatty acids and mental development in term infants. Dev Med Child Neurol 42, 174-181.

15. Ben XM, Zhou XY, Zhao WH, et al. (2004) Growth and development of term infants fed with milk with long-chain polyunsaturated fatty acid supplementation. Chin Med $J$ (Engl) 117, 1268-1270.

16. Auestad N, Scott DT, Janowsky JS, et al. (2003) Visual, cognitive, and language assessments at 39 months: a follow-up study of children fed formulas containing long-chain polyunsaturated fatty acids to 1 year of age. Pediatrics $\mathbf{1 1 2}$, e177-e183. 
17. Auestad N, Halter R, Hall RT, et al. (2001) Growth and development in term infants fed long-chain polyunsaturated fatty acids: a double-masked, randomized, parallel, prospective, multivariate study. Pediatrics 108, 372-381.

18. Agostoni C, Trojan S, Bellu R, et al. (1997) Developmental quotient at 24 months and fatty acid composition of diet in early infants: a follow up study. Arch Dis Child 76, 421-424.

19. Agostoni C, Riva E, Trojan S, et al. (1995) Docosahexaenoic acid status and developmental quotient of healthy term infants. Lancet 346, 638.

20. Agostoni C, Zuccotti GV, Radaelli G, et al. (2009) Docosahexaenoic acid supplementation and time at achievement of gross motor milestones in healthy infants: a randomized, prospective, double-blind, placebo-controlled trial. $\mathrm{Am}$ J Clin Nutr 89, 64-70.

21. Auestad N, Montalto MB, Hall RT, et al. (1997) Visual acuity, erythrocyte fatty acid composition, and growth in term infants fed formulas with long chain polyunsaturated fatty acids for one year. Ross Pediatric Lipid Study. Pediatr Res 41, $1-10$.

22. Birch EE, Castaneda YS, Wheaton DH, et al. (2005) Visual maturation of term infants fed long-chain polyunsaturated fatty acid-supplemented or control formula for $12 \mathrm{mo}$. Am J Clin Nutr 81, 871-879.

23. Birch EE, Carlson SE, Hoffman DR, et al. (2010) The DIAMOND (DHA Intake And Measurement Of Neural Development) Study: a double-masked, randomized controlled clinical trial of the maturation of infant visual acuity as a function of the dietary level of docosahexaenoic acid. Am J Clin Nutr 91, 848-859.

24. Bouwstra H, Dijck-Brouwer DA, Boehm G, et al. (2005) Long-chain polyunsaturated fatty acids and neurological developmental outcome at 18 months in healthy term infants. Acta Paediatr 94, 26-32.

25. Carlson SE, Ford AJ, Werkman SH, et al. (1996) Visual acuity and fatty acid status of term infants fed human milk and formulas with and without docosahexaenoate and arachidonate from egg yolk lecithin. Pediatr Res 39, 882-888.

26. Makrides M, Neumann MA, Simmer K, et al. (2000) A critical appraisal of the role of dietary long-chain polyunsaturated fatty acids on neural indices of term infants: a randomized, controlled trial. Pediatrics 105, 32-38.

27. Lucas A, Stafford M, Morley R, et al. (1999) Efficacy and safety of long-chain polyunsaturated fatty acid supplementation of infant-formula milk: a randomised trial. Lancet 354, 1948-1954.

28. Beyerlein A, Hadders-Algra M, Kennedy K, et al. (2010) Infant formula supplementation with long-chain polyunsaturated fatty acids has no effect on Bayley developmental scores at 18 months of age-IPD meta-analysis of 4 large clinical trials. J Pediatr Gastroenterol Nutr 50, 79-84.

29. Helland IB, Smith L, Saarem K, et al. (2003) Maternal supplementation with very-long-chain $n-3$ fatty acids during pregnancy and lactation augments children's IQ at 4 years of age. Pediatrics 111, e39-e44.

30. Gibson RA, Neumann MA \& Makrides M (1997) Effect of increasing breast milk docosahexaenoic acid on plasma and erythrocyte phospholipid fatty acids and neural indices of exclusively breast fed infants. Eur J Clin Nutr 51, $578-584$.

31. Lauritzen L, Jorgensen MH, Olsen SF, et al. (2005) Maternal fish oil supplementation in lactation: effect on developmental outcome in breast-fed infants. Reprod Nutr Dev 45, 535-547.

32. Jensen CL, Voigt RG, Prager TC, et al. (2005) Effects of maternal docosahexaenoic acid intake on visual function and neurodevelopment in breastfed term infants. Am J Clin Nutr 82, 125-132.

33. Simmer K \& Patole SK (2008) Longchain polyunsaturated fatty acid supplementation in infants born at term. The Cochrane Database Systematic Reviews issue 1, CD000376.

34. Hoffman DR, Boettcher JA \& Diersen-Schade DA (2009) Toward optimizing vision and cognition in term infants by dietary docosahexaenoic and arachidonic acid supplementation: a review of randomized controlled trials. Prostaglandins Leukot Essent Fatty Acids 81, 151-158.

35. Brenna JT, Varamini B, Jensen RG, et al. (2007) Docosahexaenoic and arachidonic acid concentrations in human breast milk worldwide. Am J Clin Nutr 85, 1457-1464.

36. Carlson SE (2000) Behavioral methods used in the study of long-chain polyunsaturated fatty acid nutrition in primate infants. Am J Clin Nutr 71, 268S-274S.

37. Meldrum SJ, D'Vaz N, Dunstan J, et al. (2011) The Infant Fish Oil Supplementation Study (IFOS): design and research protocol of a double-blind, randomised controlled n-3 LCPUFA intervention trial in term infants. Contemp Clin Trials 32, 771-778.

38. Litonjua AA, Carey VJ, Burge HA, et al. (1998) Parental history and the risk for childhood asthma. Does mother confer more risk than father? Am J Respir Crit Care Med 158, 176-181.

39. Fekete K, Marosvolgyi T, Jakobik V, et al. (2009) Methods of assessment of $n-3$ long-chain polyunsaturated fatty acid status in humans: a systematic review. Am J Clin Nutr 89, 2070S-2084S.

40. Mori TA, Burke V, Puddey IB, et al. (2000) Purified eicosapentaenoic and docosahexaenoic acids have differential effects on serum lipids and lipoproteins, LDL particle size, glucose, and insulin in mildly hyperlipidemic men. $A m J$ Clin Nutr 71, 1085-1094.

41. Dunstan JA, Roper J, Mitoulas L, et al. (2004) The effect of supplementation with fish oil during pregnancy on breast milk immunoglobulin A, soluble CD14, cytokine levels and fatty acid composition. Clin Exp Allergy 34, 1237-1242.

42. Makrides M, Neumann MA, Simmer K, et al. (1995) Erythrocyte fatty acids of term infants fed either breast milk, standard formula, or formula supplemented with long-chain polyunsaturates. Lipids 30, 941-948.

43. Fenson L, Dale PS, Reznik JS, et al. (1993) The MacArthur Communicative Development Inventories: User's Guide and Technical Manual. San Diego, CA: Singular Publishing Group.

44. Bayley N (2006) Bayley Scales of Infant and Toddler Development, 3rd ed. San Antonio, TX: Harcourt Assessment.

45. Achenbach TM \& Rescola LA (2000) Manual for the ASEBA Preschool Forms and Profiles. Burlington, VT: University of Vermont Department of Psychiatry.

46. Makrides M, Neumann MA, Simmer K, et al. (1999) Dietary long-chain polyunsaturated fatty acids do not influence growth of term infants: a randomized clinical trial. Pediatrics 104, 468-475.

47. Dunstan JA, Mitoulas LR, Dixon G, et al. (2007) The effects of fish oil supplementation in pregnancy on breast milk fatty acid composition over the course of lactation: a randomized controlled trial. Pediatr Res 62, 689-694.

48. Goodwyn SW \& Acredolo LP (1998) Encouraging symbolic gestures: a new perspective on the relationship between gesture and speech. New Dir Child Dev 61-73.

49. Innis SM, Gilley J \& Werker J (2001) Are human milk longchain polyunsaturated fatty acids related to visual and neural development in breast-fed term infants? J Pediatr 139, 532-538. 
50. Hallahan B \& Garland MR (2005) Essential fatty acids and mental health. Br J Psychiatry 186, 275-277.

51. Peet M \& Stokes C (2005) Omega-3 fatty acids in the treatment of psychiatric disorders. Drugs 65, 1051-1059.

52. Neisworth JT \& Bagnato SJ (2004) The mismeasure of young children: the authentic assessment alternative. Infant Young Child 17, 198-212.

53. Cheatham CL, Colombo J \& Carlson SE (2006) $n$-3 Fatty acids and cognitive and visual acuity development: methodologic and conceptual considerations. Am J Clin Nutr $\mathbf{8 3}$, $1458 \mathrm{~S}-1466 \mathrm{~S}$

54. Prescott SL, Macaubas C, Holt BJ, et al. (1998) Transplacental priming of the human immune system to environmental allergens: universal skewing of initial $\mathrm{T}$ cell responses toward the Th2 cytokine profile. J Immunol 160, 4730-4737.

55. Chida Y, Hamer M \& Steptoe A (2008) A bidirectional relationship between psychosocial factors and atopic disorders: a systematic review and meta-analysis. Psychosom Med 70, 102-116.

56. Liu CC, Carlson SE, Rhodes PG, et al. (1987) Increase in plasma phospholipid docosahexaenoic and eicosapentaenoic acids as a reflection of their intake and mode of administration. Pediatr Res 22, 292-296.

57. Nordoy A, Barstad L, Connor WE, et al. (1991) Absorption of the $n-3$ eicosapentaenoic and docosahexaenoic acids as ethyl esters and triglycerides by humans. Am J Clin Nutr 53, 1185-1190.

58. Sala-Vila A, Castellote AI, Campoy C, et al. (2004) The source of long-chain PUFA in formula supplements does not affect the fatty acid composition of plasma lipids in full-term infants. J Nutr 134, 868-873

59. Commonwealth Scientific and Industrial Research Organisation (1996) CSIRO Australian Food and Nutrition Surveys 1988 and 1993: Results from the CSIRO Australian Food and Nutrition Surveys 1988 and 1993. Adelaide, SA: CSIRO Division of Human Nutrition.

60. Cramer JA, Scheyer RD \& Mattson RH (1990) Compliance declines between clinic visits. Arch Intern Med 150, 1509-1510

61. Bacchetti P (2002) Peer review of statistics in medical research: the other problem. BMJ 324, 1271-1273. 